Pecori eine Reihe von Eigentümlichkeiten und knüpft interessante Betrachtungen daran. A. verweilt besonders bei dem Trichophyton cerebriforme, Tr. gypseum asteroides, Tr. rosaceum, Tr. faviforme (Ochraceum).

Das Problem der Unterscheidung der Trichophytien in Ektothrix- und Endothrixformen, in torpide und entzündliche Formen, der Korrespondenz zwischen einer bestimmten klinischen Form und einer bestimmten Trichophytonart, wird von A. diskutiert.

Bemerkenswert ist, daß A. in Rom niemals Mikrosporien durch Mikrosporon Audouini beobachtet hat.

Die ansführliche und fleißige Arbeit $\mathrm{P}$ e cor is wird allen, die sich für dieses Gebiet interessieren, eine Reihe nützlicher Kenntnisse vermitteln können. J. Ullman n (Rom).

\title{
Polnische Zeitschrift für Haut- und venerische Krankheiten.
}

(Przegląd chorób skórnych i wener. 1913. Bd. VIII. H. 1, 2, 3.)

Kraysztalowicz, F. Histologie des Keratoma palmare et plantare hereditarium.

Heutzutage wird bei Beurteilung der pathologischen Zustände in dem Verhornungsprozesse die Lagerung und die Dicke der Hornmassen und das Dasein der kernhaltigen Hornzellen oder Fehlen derselben meistens berücksichtigt, diese Bilder genügen jedoch nicht, um dem Wesen der pathologischen..Verhornung näher zu treten. Der Verf. hat daher auch die Übergangsschichten, ihre Lagerung und Entwicklung näher studiert, um einen Schluß zu ermöglichen, auf welche Weise die dickeren Hornmassen hier zur Entwicklung gelangten. $\mathrm{Zu}$ diesem weitgehenden Zwecke wurden die durch Unna und Golodetz in der letzten Zeit angegebenen Reaktions- und spezifischen Färbungen benutzt und auf Grund dieser Untersuchungen ist K. zu. dem Schlusse gekommen, daß bei dem Keratoma hereditarium eive starke Hyperplasie des Epithels mit besonderer Entwicklung der Hornmassen zustande kommt. Die breite mächtige Körnersehicht und die folgende auch breite glykogenhaltige Schicht, wie auch die Entwicklungsart der basalen Hornschicht führt zu dem Schlusse, daß die stärker sich entwickelnden Stachelzellen schneller dem Verhornungsprozesse unterliegen, als dies im normalen Zustande der Fall ist. Die eleïdinhaltige Zellenschicht scheint der Körnerschicht proportional $\mathrm{zu}$ sein und die verhornten Zellenreihen sind auch viel dicker, als es der normalen Fußsohle entsprechen könnte. Die an Albumosen und Keratin reichen Hornzellen sind in einer so 
regelmäßigen Anordnung in den Wellentälern und Bergen, wie normal, nicht zu finden. Der Verhornungsprozeß scheint sich hier also in etwas beschleunigter und unregelmäBiger Weise, dennoch aber sonst normal, zu vollziehen.

Serloowski, St. Vakz in et herapie. (Fortsetzung.)

Die weitere kritische Besprechung der Wrightschen Theorie, ihre Bedeutung in der Pathologie und Therapie und eigene Versuche des Verfassers in dieser Richtung.

Wiśniewski, J. Parapsorias is Brocq.

Der Verf. beschreibt einen Fall ron Parapsoriasis en gouttes (Typus Jadassohn) bei einem 12jährigen schwach entwickelten und schlecht ernährten Mädchen, welche an den oberen Extremitäten (Extensorenseite) zutage trat. Das Exanthem bestand aus kleinen, unregelmäßigen Flecken und Papeln, die stecknadelkopfgroß und mit grauen silberglänzenden Schuppen bedeckt waren. Die mikroskopische Untersuchung scheint diese Diagnose zu bestätigen, F. Krzysztalowicz (Krakau).

\title{
Dermatologie.
}

\author{
Bd. I. H. 6. Juni 1913 .
}

1. Sehiperskaya, A. K. Zur Frage der Pseudofurunkulosis (Finger). p. 559.

Hauptsächlich bei Säuglingen kommt eine Pyodermie in Form eitrig zerfallender Knötchen und Knoten vor, wobei kein Zusammenhang mit Haarbälgen und Talgdrüsen nachzuweisen ist. Die Krankheit führt zur Genesung oder zur Pyämie. Die Verfasserin beobachtete 9 Fälle im Alter von 2-6 Monaten. Nur in einem Falle handelte es sich um einen Erwachsenen (20 J.): In 5 Fällen wurde Staphylococcus aureus, in 5 Staphylococcus alb. gezüchtet. In 6 Fällen fiel die Agglutination positiv aus. In 5 Fällen (Staphylokokken im Blute) kam es zum Exitus; bei der Autopsie wurden lobuläre chronische Pneumonie, chronische Enteritis, Fettdegeneration des Herzens, der Leber und Nieren, Tumor lienis gefunden. Histologisch konnte nur bei 2 Kranken Periporitis konstatiert werden. Schwere Fälle sind also infolge hämatogener Infektion entstanden, in leichten ist aber die Pathogenese unklar (entweder hämatogene oder exogene Infektion?).

2. Milmann, J. S. Zur Lehre von der Parap soriasis en plaques (Brocq). p. 565.

Ein 25jähriger Schutzmann zeigt seit 4 Monaten am Halse, Abdomen, in der Inguinal- und Sakralregion, sowie an Oberschenkeln einen Ausschlag, der aus runden und oval-ellyptischen, $3-4 \mathrm{~cm}$ großen, gelblich-roten, schwach schuppende, nicht infiltrierten Flecken besteht. Keine subjektiven Beschwerden. 